\title{
Modeling the signature of a transponder in altimeter return data and determination of the reflection surface of the ice cap near the GRIP camp, Greenland
}

\author{
G. Haardeng-Pedersen, ${ }^{1}$ K. Keller, ${ }^{2}$ C. C. Tscherning, ${ }^{2}$ N. Gundestrup ${ }^{2}$ \\ ${ }^{1}$ Physics Department, Memorial University of Newfoundland (SWGC), Corner Brook, Newfoundland A2H 6P9, Canada \\ ${ }^{2}$ Department of Geophysics, University of Copenhagen, DK-2100 Copenhagen O, Denmark
}

\begin{abstract}
Using an active transponder with the ERS-1 and ERS-2 radar altimeters, the distance to the satellite was measured at a location close to the GRIP site, Greenland, at an altitude of $3.2 \mathrm{~km}$. The measurement was executed while the transponder was in the "ice-tracking mode". It includes a bias due to the propagation delay. The location of the transponder was determined using the global positioning system.

The transponder signal was modeled and the distance from the altimeter to the effective reflection point of the transponder was determined. Since the transponder was located within $1 \mathrm{~km}$ of the ground tracks, the measurement was corrected for this offset. A correction was also done for the surface slope, resulting in the distance (plus bias) to the closest sub-satellite point on the surface of the (compact) snow.

The transponder signal was then removed from the radar altimeter waveform, enabling the determination of the distance (plus bias) from the altimeter to the first reflective surface within the snow. The difference between this distance and that obtained using the transponder was $<2 \mathrm{~m}$. This shows that the surface which gives rise to the first return of the reflection agrees with the surface of the (compact, dry) snow at this high-altitude location. This is an important result to be used when studying ice-cap topography using satellite radar altimetry.
\end{abstract}

\section{INTRODUGTION}

As part of the GRIP (Greenland Ice Core Project)-ERS-1 (European Remote-sensing Satellite 1) calibration on the Greenland ice cap (Keller and others, 1994) a ground-based active transponder (Powell, 1986; Powell and others, 1993) was deployed on the Greenland ice cap. The transponder receives, amplifies and retransmits the radar pulses from the radar altimeter on ERS-1. Two successful trials had been accomplished in Jutland, Denmark, in spring 1993. Five attempts were then made with the transponder to acquire and retransmit the altimeter signal in Greenland, three at Saddle North in June 1993 and two near the GRIP ice-core drilling site at the top of the Greenland ice cap in July 1993 (Fig. 1). These sites were carefully selected to be at locations with very low surface slopes, minimal sastrugi and dry, cold snow, thus providing optimal conditions for the radar altimeter. Only one of the experiments, near the GRIP site, was successful; the other four attempts succumbed to a blizzard, a temporary shut-down of the altimeter or minor technical problems that were subsequently corrected. In 1995, two transponder experiments were successful; both were located near the GRIP site, one using the ERS-1 satellite, the other using the ERS-2 satellite. These experiments, and a detailed knowledge of the topography of the ice cap in the area around GRIP (Ekholm and Keller, 1993; Forsberg, 1996), have allowed us to determine the depth within the snow from which the radar pulse is first reflected.

Experiments are planned that will extend this analysis to other parts of the Greenland ice cap, where the dielectric properties of the snow may differ, for example because of temperature (Jezek and others, 1994). The transponder will then provide a ground-truth measurement for the radar altimeter's determination of the topography of the ice cap. Repeated altimeter measurements of the ice-sheet surface elevation can then be used as a signal to monitor temporal changes due to any "greenhouse effect".

\section{RETURN TIME OF A PULSE IN A SERIES OF SPIKE PULSES}

The transponder acts as a point reflector (with amplification) whereby the ice sheet can be modeled (Brown, 1977) as a rough, flat surface.

The geometry of the configuration used to derive the return time of one of the pulses emitted by the altimeter is shown in Figure 2. We have selected a reference plane that is the orbital plane of the satellite as viewed from the Earth.

The transponder is a "portable" instrument (weighing $85 \mathrm{~kg}$ ) that can be transported to a site; optimally it will be located at a point on the anticipated ground track below the satellite. We assume that the orbit of the satellite is circular, of radius $S=R+h$, that the transponder lies in this plane, a distance $R$ from the center of the Earth - so that the altimeter passes through the zenith point of the transponder a distance $h$ above the transponder location - and that the speed of the altimeter (relative to the transponder) is $v$.

The altimeter emits radar pulses at regular intervals $T$ $=9.804 \times 10^{-4} \mathrm{~s}$. For simplicity, we initially assume that these 


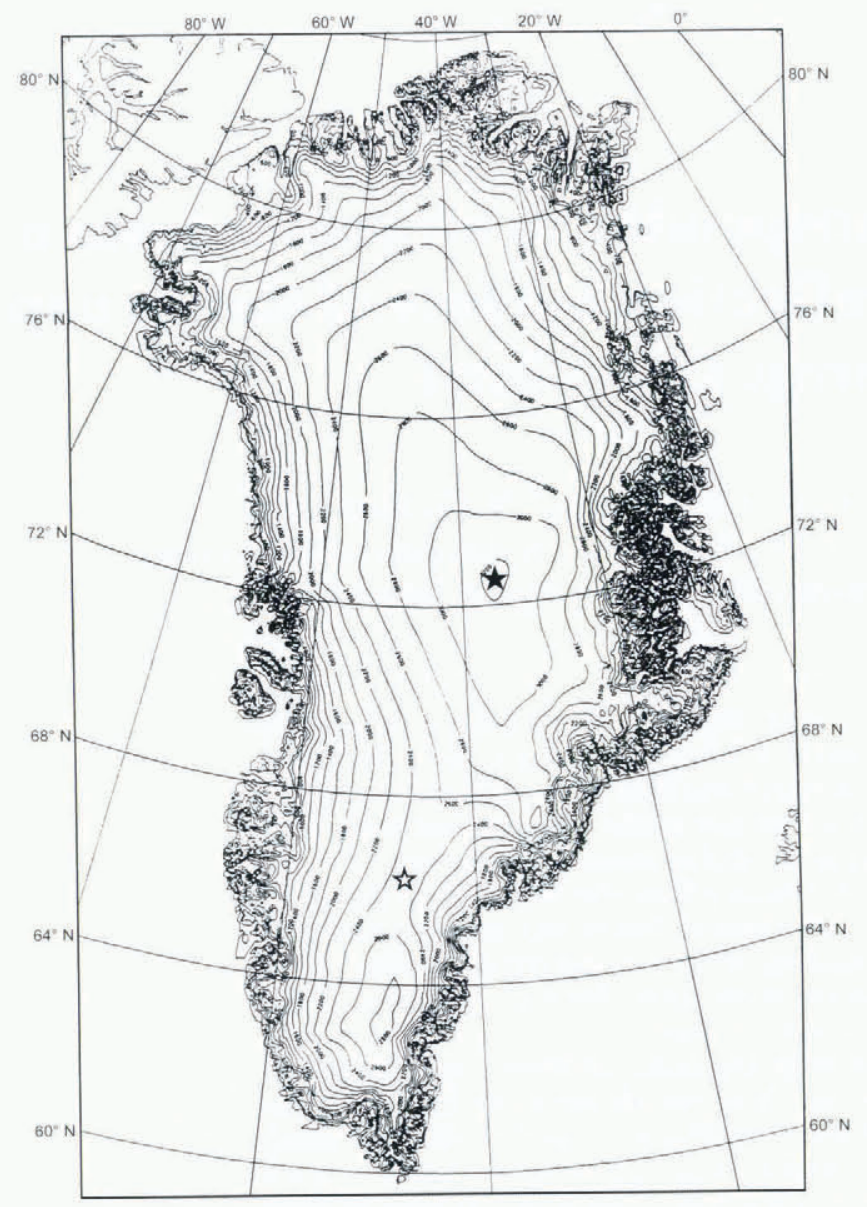

Fig. 1. The two locations on the Greenland ice sheet where the transponder was deployed. The GRIP site $\left(72.45^{\circ} \mathrm{N}\right.$, $\left.36.2^{\circ} \mathrm{W}\right)$ is indicated by the solid star, and Saddle North $\left(66.45^{\circ} \mathrm{N}, 43.3^{\circ} \mathrm{W}\right)$ by the open star.

pulses are spikes (delta functions) and, for convenience, that one of these pulses is emitted at the instant that the satellite passes through the zenith position. This pulse is then labeled as pulse zero, preceding pulses labeled with positive integer values $(1,2,3, \ldots)$ and pulses emitted after the altimeter has passed through the zenith point labeled with negative integer values $(-1,-2,-3, \ldots)$.

At the instant that the altimeter is emitting the pulse labeled $n$, it is at a position which is a distance $n v T$ along the orbital arc from the zenith position. The distance that this pulse travels to the altimeter is

$$
d_{1}=\sqrt{S^{2}+R^{2}-2 S R \cos \theta_{n}}=c t_{1} .
$$

Here $\theta_{n}$ is the angle subtended at the center of the Earth by the arc from the satellite's current position to the zenith position, and $t_{1}$ is the one-way travel time from the altimeter to the transponder. In radians, the angle $\theta_{n}$ is $n v T / S$. The return pulse from the transponder travels for a time $t_{2}$, intercepting the altimeter at the instant that it is a distance $v\left(n T-t_{1}-t_{2}\right)$ from the zenith position. At this instant the angle subtended at the center of the Earth by the arc from the satellite position to the zenith position is $\theta_{n}^{\prime}=v\left(n T-t_{1}-t_{2}\right) / S$, and the distance that the return pulse travels is

$$
d_{2}=c t_{2}=\sqrt{S^{2}+R^{2}-2 S R \cos \theta_{n}^{\prime}} .
$$

The total travel time of this pulse, to the transponder and back, is $\tau_{n}=t_{1}+t_{2}$ so that $\theta_{n}^{\prime}=\theta_{n}-\left(v \tau_{n}\right) / S$, and the distance traveled by the pulse is

$$
\begin{aligned}
c \tau_{n} & =\sqrt{S^{2}+R^{2}-2 S R \cos \theta_{n}} \\
& +\sqrt{S^{2}+R^{2}-2 S R \cos \theta_{n}^{\prime}} \\
& =\sqrt{h^{2}+2 R S\left(1-\cos \theta_{n}\right)} \\
& +\sqrt{h^{2}+2 R S\left[1-\cos \left(\theta_{n}-\frac{v \tau_{n}}{S}\right)\right]} .
\end{aligned}
$$

Rewriting this as

$$
\begin{aligned}
c \tau_{n} & -\sqrt{h^{2}+2 R S\left(1-\cos \theta_{n}\right)} \\
& =\sqrt{h^{2}+2 R S\left[1-\cos \left(\theta_{n}-\frac{v \tau_{n}}{S}\right)\right]},
\end{aligned}
$$

squaring both sides and simplifying leads to

$$
\begin{aligned}
c^{2} \tau_{n}{ }^{2} & =2 c \tau_{n} \sqrt{h^{2}+2 R S\left(1-\cos \theta_{n}\right)} \\
& +2 R S\left[\cos \theta_{n}-\cos \left(\theta_{n}-\frac{v \tau_{n}}{S}\right)\right]
\end{aligned}
$$

and then

$$
\begin{aligned}
\tau_{n} & =\frac{2 h}{c} \sqrt{1+\frac{2 R S}{h^{2}}\left(1-\cos \theta_{n}\right)} \\
& +\frac{2 R S}{c^{2}} \frac{\cos \theta_{n}-\cos \left(\theta_{n}-\frac{v \tau_{n}}{S}\right)}{\tau_{n}}
\end{aligned}
$$

We note that the second term is a small correction to the first term and that an iterative technique converges quickly to determine $\tau_{n}$.

It is a worthwhile exercise at this point to develop an expansion of $\tau_{n}$ as a power series in $n$ to show the character of the transponder return.

The first term can be written as

$$
\begin{aligned}
& \frac{2 h}{c}\left[1+\frac{2 R S}{h^{2}}\left(1-\cos \theta_{n}\right)\right]^{\frac{1}{2}} \\
& \quad \approx \frac{2 h}{c}\left[1+\frac{1}{2}\left(1-\cos \theta_{n}\right)-\frac{1}{8}\left(1-\cos \theta_{n}\right)^{2}+\ldots\right],
\end{aligned}
$$

and since

$$
1-\cos \theta_{n}=\frac{1}{2}\left(\frac{n v T}{S}\right)^{2}-\frac{1}{8}\left(\frac{n v T}{S}\right)^{4}+\ldots
$$

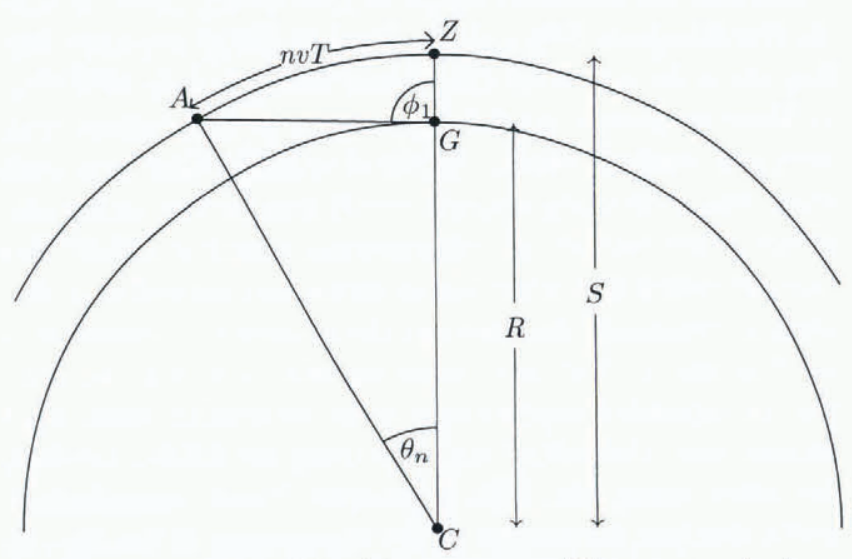

Fig. 2. Geometry of the altimeter passage. The transponder is deployed at position $G$, a distance $R$ from the center of the Earth, $C$. The altimeter is a distance $S$ from the center of the Earth, traveling at a constant speed $v$; it emits a pulse at $A$ at a time $n T$ before it passes through the zenith position $Z$. 
the leading terms of the power-series expansion are then

$$
\frac{2 h}{c}+\frac{R}{S} \frac{v^{2} T^{2}}{h c} n^{2}-\frac{R}{12 S}\left(\frac{1}{S^{2}}+\frac{3}{h^{2}}\right) \frac{v^{4} T^{4}}{h c} n^{4} .
$$

The second term in the expression for $\tau_{n}$ can be rewritten as

$$
\begin{aligned}
& -\frac{2 R S}{c^{2}} \frac{2}{\tau_{n}} \sin \left(\frac{v \tau_{n}}{2 S}\right) \sin \left(\theta_{n}-\frac{v \tau_{n}}{2 S}\right) \\
& \approx \frac{R v^{2}}{S c^{2}} \tau_{n}-\left(\frac{2 R v^{2}}{S c^{2}} T\right) n+\left(\frac{R v^{4} T^{3}}{3 S^{3} c^{2}}\right) n^{3} .
\end{aligned}
$$

Gathering these expansions together, the power-series expansion of $\tau_{n}$ complete to order $n^{4}$ is

$$
\begin{gathered}
\tau_{n}\left(1-\frac{R v^{2}}{S c^{2}}\right)=\frac{2 h}{c}-\left(\frac{2 R v^{2}}{S c^{2}} T\right) n+\frac{R}{S} \frac{v^{2} T^{2}}{h c} n^{2} \\
+\left(\frac{R v^{4} T^{3}}{3 S^{3} c^{2}}\right) n^{3}-\frac{R}{12 S}\left(\frac{1}{S^{2}}+\frac{3}{h^{2}}\right) \frac{v^{4} T^{4}}{h c} n^{4} .
\end{gathered}
$$

For the ERS-1 satellite, the numerical values of the quantities used above are:

$$
\begin{aligned}
R & \approx 6.37 \times 10^{6} \mathrm{~m} \\
S & \approx 7.16 \times 10^{6} \mathrm{~m} \\
v & \approx 7.50 \times 10^{3} \mathrm{~m} \mathrm{~s}^{-1} \\
c & =3.00 \times 10^{8} \mathrm{~m} \mathrm{~s}^{-1} \\
h & \approx 7.925 \times 10^{5} \mathrm{~m} \\
T & =9.804 \times 10^{-4} \mathrm{~s}
\end{aligned}
$$

Substituting these values into the power-series expansion leads to an estimate of the return time $\tau_{n}(\mathrm{~s})$ as

$$
\begin{aligned}
\tau_{n} & \approx\left(5.3 \times 10^{-3}\right)-\left(1.25 \times 10^{-12} n\right)+\left(0.20 \times 10^{-12} n^{2}\right) \\
& +\left(2 \times 10^{-25} n^{3}\right)-\left(5 \times 10^{-24} n^{4}\right),
\end{aligned}
$$

and for the transponder return to be visible in the altimeter record $|n| \leq 2000$, and this shows that the variation in the travel time of the pulse as the altimeter passes over the transponder is, to a good approximation, a parabolic function of the distance from the altimeter to the zenith point of the transponder. It is this unique pattern (along with the amplification of the signal by the transponder) that makes the transponder return very easy to identify in the altimeter record.

We choose to compute the delays in these travel times relative to the delay of the pulse emitted at the instant that the altimeter is at the zenith position, i.e. the pulse labeled $n=0$. The travel time for this pulse is

$$
\tau_{0}=\frac{2 h}{c}+\frac{2 R S}{c^{2}}\left[\frac{1-\cos \left(\frac{v \tau_{0}}{S}\right)}{\tau_{0}}\right] .
$$

The additional delay, $\Delta_{n}$, of the pulse emitted at time $n T$ before the altimeter reaches the zenith position is

$$
\Delta_{n}=\tau_{n}-\tau_{0},
$$

and from Equation (2) this can be written (so as to retain as much accuracy as possible in the computations) as:

$$
\begin{aligned}
& \Delta_{n}=\frac{2 h}{c}\left[\sqrt{1+\frac{2 R S}{h^{2}}\left(1-\cos \theta_{n}\right)}-1\right] \\
& +\frac{2 R S}{c^{2}}\left[\frac{\cos \theta_{n}-\cos \left(\theta_{n}-\frac{v \tau_{n}}{S}\right)}{\tau_{n}}-\frac{1-\cos \left(\frac{v \tau_{0}}{S}\right)}{\tau_{0}}\right] .
\end{aligned}
$$

From this specification a set of values of the relative delays can be computed for a specified pair of the parameters $h$ and $v$.

\section{AMPLITUDE VARIATION OF RETURNED PULSE}

The pulses are highly directional; the beamwidth, $W$, is $1.36^{\circ}=0.02374 \mathrm{rad}$. For simplicity we assume that the altimeter passes directly over the transponder, that the antenna lies in the plane of the orbit and that the boresight of the antenna points at the center of the Earth. We neglect any variation in the signal strength due to the small changes in distance between the altimeter and the transponder.

The gain for each path between the altimeter and the transponder, when the angle between the boresight of the antenna and the antenna-transponder line is $\phi$, is

$$
g(\phi)=\exp \left[-2 \ln 4\left(\frac{\phi}{W}\right)^{2}\right]
$$

For the pulse from the altimeter to the transponder, the arc from the altimeter to the zenith point has a length of $n v T$, the angle subtended by this arc at the Earth's center is $\theta_{n}$, and the angle subtended by this arc at the transponder's location is $\psi=n v T / h$. Referring to Figure 2, the angle $\phi_{1}$ for the forward pulse is then

$$
\phi_{1}=\psi-\theta_{n}=\frac{n v T}{h}-\frac{n v T}{S}=\frac{n v T}{h} \frac{R}{S} .
$$

The same gain occurs on the return path except that the distance from the zenith point to the altimeter's position has become $n v T-v \tau_{n}$. The appropriate angle for the return path is

$$
\phi_{2}=\frac{v\left(n T-\tau_{n}\right)}{h} \frac{R}{S} .
$$

By setting

$$
w^{2}=\frac{h^{2} W^{2} R^{2}}{2 \ln 4 v^{2} T^{2} S^{2}}
$$

and approximating $\tau_{n}$ by $\tau_{0}$, the total gain on the pulse labeled $n$ is

$$
G_{n}=\exp \left(\frac{-n^{2}}{w^{2}}\right) \exp \left[\frac{-(n-\gamma)^{2}}{w^{2}}\right]
$$

where $\gamma=\tau_{0} / T$.

If there is a small along-track pointing error in the boresight of the altimeter, the maximum amplitude of the returned pulse would not coincide with the shortest return time of the pulse, i.e. if the antenna of the altimeter points slightly ahead of vertically downward, the maximum amplitude of the returned pulse would occur before the satellite reaches the zenith position, at some positive value of $n$ rather than at $n=0$. An equivalent offset would occur when the transponder, which is leveled by a spirit level, is at a small tilt. A small tilt of the transponder towards the incoming altimeter would provide the same offset of the maximum amplitude of the returned pulse to a positive value of $n$.

The possibility of either offset can be taken into account in the modeling of the amplitude variation of the pulse in a simple way by introducing a parameter $N$, the value of $n$ at which the gain is maximum, and then revising the gain function to

$$
G_{n}=\exp \left[\frac{-(n-N)^{2}}{w^{2}}\right] \exp \left[\frac{-(n-N-\gamma)^{2}}{w^{2}}\right] .
$$




\section{DIVISION OF THE PULSE INTO BINS, AND THE SHAPE OF THE WAVEFORM GENERATED}

Until this section, the altimeter pulse (and the reflected pulse) have been assumed to be delta functions or spikes. However, the point target response of the altimeter radar should be modeled as a Gaussian return, distributed around the arrival time of the spike.

Instead of a delta function response after a lag time of $\tau_{n}$ for the pulse labeled $n$, the appropriate model is then a response:

$$
r_{n}(t)=A_{n} \exp \left[-\frac{\left(t-\tau_{n}\right)^{2}}{2 \sigma^{2}}\right]
$$

where the parameter $\sigma$ is $6.604150 \mathrm{~ns}$ for the ice mode of the altimeter, and the pulse amplitude $A_{n}$ is the product of a constant $A_{\max }$ (itself determined by the power in the original transmission from the altimeter and the amplification produced by the transponder) and the gain of this individual pulse, $G_{n}$, due to the bandwidth.

The altimeter, after emitting a pulse, waits for a time this waiting interval is determined in part by the recent history of its receptions - and then begins to record the returned signal. The altimeter computer records the signal strength at the start of 64 consecutive time intervals. The duration of each of these intervals (one bin) is $B$; in the ice mode $B=12.159533 \mathrm{~ns}$. The entire 64 bins $(\approx 800 \mathrm{~ns})$ is called the range window. Normally the range window drifts as the altimeter computer updates the waiting interval with each new return; however, the waiting interval during a transponder experiment is fixed for the duration of the experiment.

We suppose that the pre-set waiting time is $\tau_{0}-X$ and then consider a sequence of bins where bin 1 starts at $\tau_{0}-X$, bin 2 starts at $\tau_{0}-X+B$, and, in general, bin $M$ starts at $\tau_{0}-X+(M-1) B$.

The response recorded for bin $M$ is then

$$
\begin{aligned}
r_{n} & {\left[\tau_{0}-X+(M-1) B\right] } \\
& =A_{n} \exp \left\{-\frac{\left[\tau_{0}-X+(M-1) B-\tau_{n}\right]^{2}}{2 \sigma^{2}}\right\} \\
& =A_{n} \exp \left\{-\frac{\left[\Delta_{n}-X+(M-1) B\right]^{2}}{2 \sigma^{2}}\right\} .
\end{aligned}
$$

Since $B /(\sigma \sqrt{2})=1.3019$, contributions from this pulse will only be significant for, at most, seven consecutive bins centered upon the bin in which the spike would appear.

The altimeter in fact accumulates the contributions to the 64 bins for 50 consecutive radar returns to produce a waveform. During the time that the altimeter passes over the transponder and is within the range at which it can acquire the radar returns, about 4000 pulses are emitted and the returns accumulated into 80 waveforms. We will call this set of 80 waveforms the observed "signature" of the transponder (Fig. 3).

The nature of the transponder return, because of the amplification of the signal by the transponder and also because of the unique structure of the set of waveforms recorded, can then be used to determine the zenith distance between the transponder and the altimeter with great precision.

Using the arguments expressed above, it is possible to generate a theoretical "signature" of the transponder. This signature will of course depend on a number of parameters,

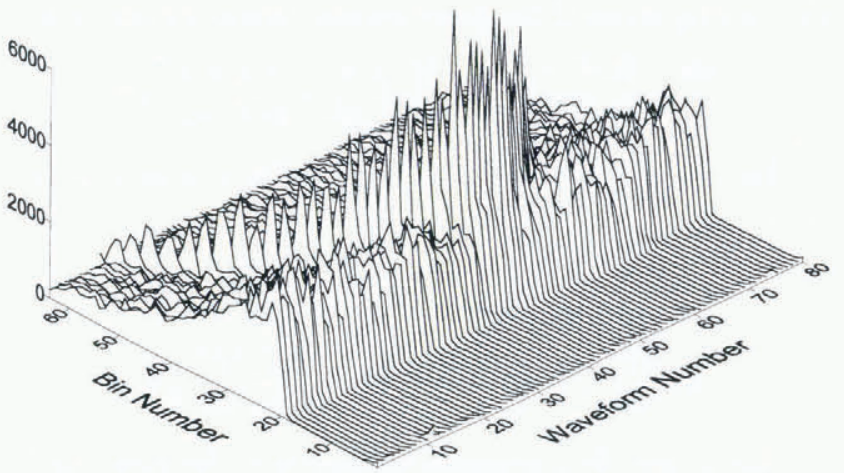

Fig. 3. The observed signature of the transponder. This is the set of 80 waveforms that were recorded as the satellite passed over the transponder's location. Note the distinctive pattern caused by the transponder; it is almost parabolic and easily visible due to the amplification.

for example the speed $v$ of the altimeter relative to the transponder, the height $h$ of the altimeter over the transponder as it passes through the zenith point, the factor $X$ in the waiting time, the pointing error $N$ in the antennae, and the maximum amplitude of the return $A_{\max }$, as well as the fixed parameters, the bin size $B$, the spread of the signal $\sigma$, and the beamwidth $W$ (or equivalently $w$ ). From the initial values selected for these parameters a set of waveforms, a theoretical signature of the transponder, can be calculated (Fig. 4).

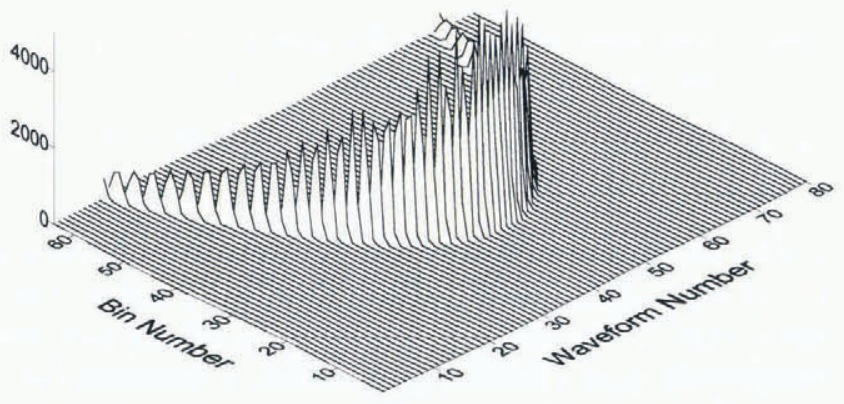

Fig. 4. The theoretical signature of the transponder.

This theoretical signature is then subtracted from the observed signature to leave a residual. Since the altimeter is sensitive only to the amplitude of the returned pulse and not to its phase, the contents of each bin in the observed signature must be non-negative and the residual therefore should have (if there were no noise in the signal) no negative values. The criterion that was used to measure the quality of the fit of the theoretical signature onto the observed signature was taken to be the sum of the positive contents of the bins in the residuals minus a penalty factor times the sum of the negative contents. If the penalty factor had been set to

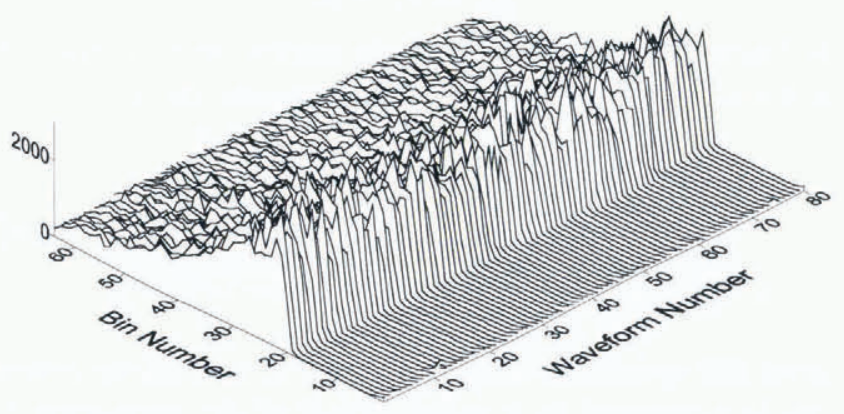

Fig. 5. The residual signature. 
unity, the quality of the fit would have been measured by the sum of the absolute values of the bin contents; however, we chose a penalty factor, $p$, of 250 , thus strongly favoring residuals where very few of the bin contents were negative.

This observed signature, $\mathcal{S}$, is an 80 by 64 matrix of integer values; each of the 80 rows is a waveform. For any chosen set of the parameters $\left(v, h, X, N\right.$ and $\left.A_{\max }\right)$ a theoretical signature, $\mathcal{T}$, also an 80 by 64 matrix of integer values, is computed. The residual signature is then the 80 by 64 matrix of integer values, $\mathcal{R}$,

$$
\mathcal{R}=\mathcal{S}-\mathcal{T} .
$$

The criterion of best fit of the theoretical signal to that observed is that

$$
C=\sum_{\substack{\text { positive } \\
\text { elements }}} \mathcal{R}_{i j}-p \sum_{\begin{array}{c}
\text { negative } \\
\text { elements }
\end{array}} \mathcal{R}_{i j}
$$

was a minimum.

The ERS-1 satellite is in an orbit with an inclination of $98.543^{\circ}$ and a semi-major axis of $7159.496 \mathrm{~km}$ (ESA, 1993). Ignoring the non-sphericity of the Earth, the initialization value of the height $h$ would be $788 \mathrm{~km}$; taking the oblateness of the Earth's figure into account, the initialization value actually used was $801 \mathrm{~km}$.

The values of $v, h, X, N$ and $A_{\max }$ are adjusted, in an iterative procedure, to calculate theoretical signatures and residuals, and the quality of each of these determined in order to leave a minimum remnant $C$ in the residual set of waveforms.

In practice, the procedure appears to be quite sensitive to the changes in these parameters, so that the values that provide the best fit of the theoretical signature to that observed are well determined (Figs 6-9).

The set of 80 waveforms that constitute the observed signature acquired on 7 July 1993, the theoretical signature which best fits this observed signature and the corresponding residual signature are shown in Figures 3-5.

Once the theoretical signature of the transponder return

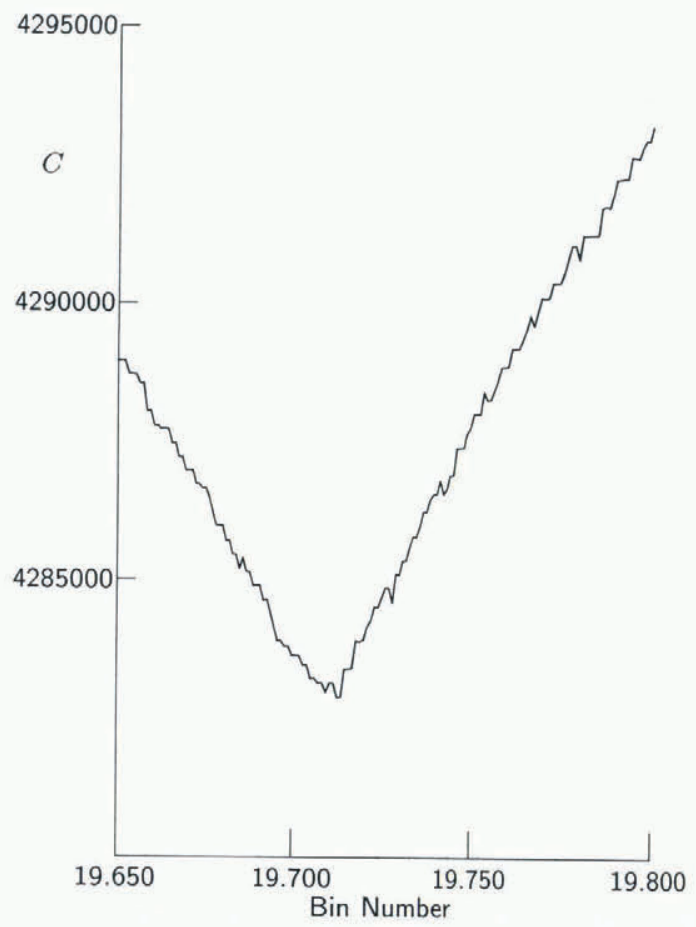

Fig. 6. Variation of the criterion, $C$, with bin number.

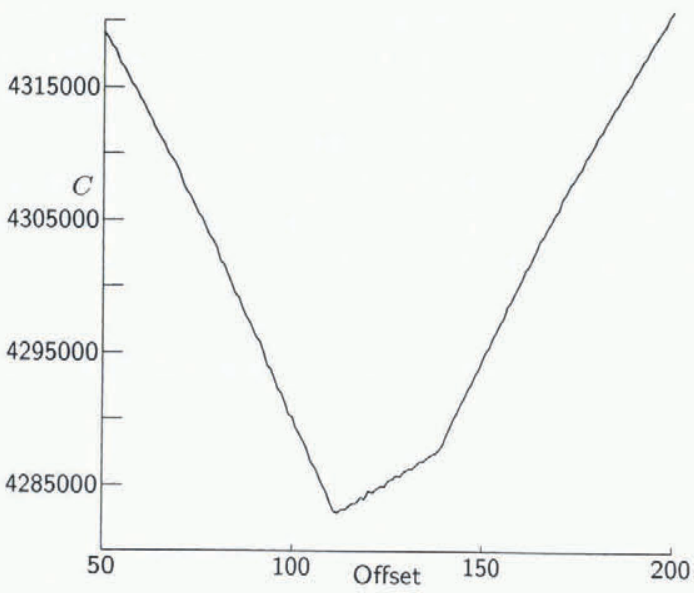

Fig. 7. Variation of the criterion, $C$, with offset number, $N$.

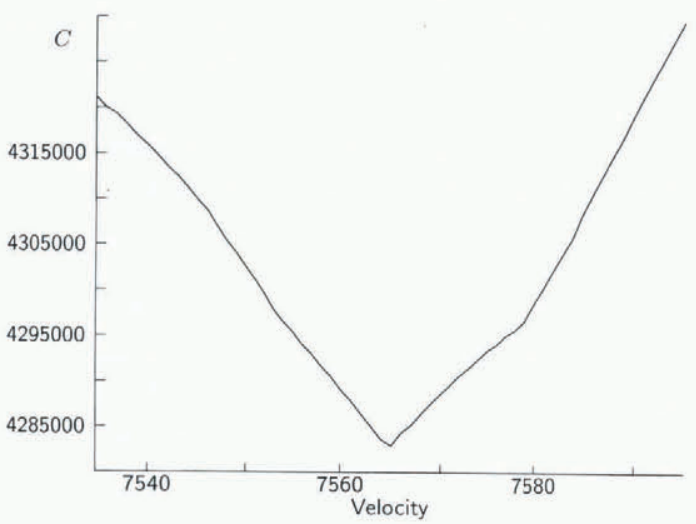

Fig. 8. Variation of the criterion, $C$, with the velocity of the altimeter relative to the transponder.

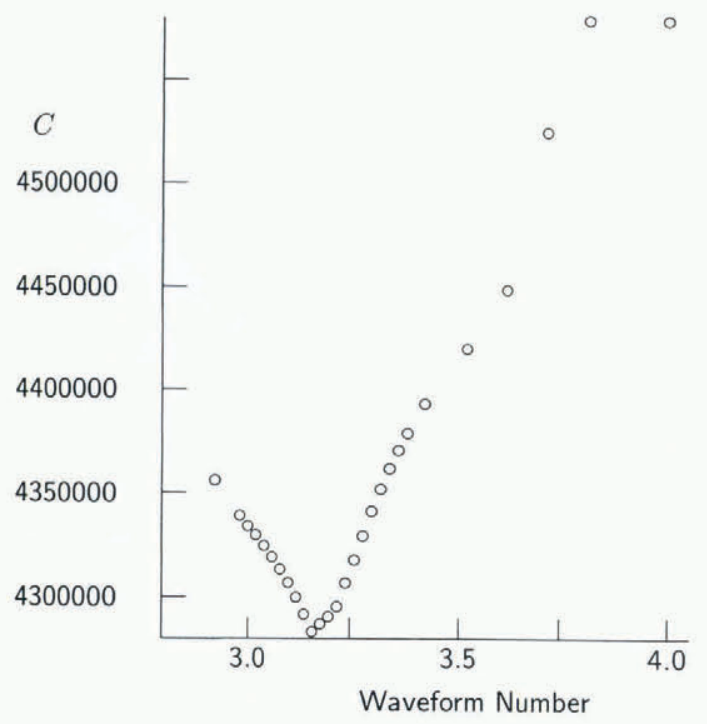

Fig. 9. Variation of the criterion, $C$, with the wavenumber at which the altimeter is at the zenith point above the transponder.

has been established using the above procedure, a parabola is then fitted to this theoretical signature to determine the zenith range to the altimeter for this overpass.

The two experiments from 30 June and 1 July 1995 also had the transponder signature clearly visible in the altimeter record. These data have been analyzed in the same manner as described above to determine the distance from the transponder location to the altimeter. Again, the smooth variation of the criterion $C$ with respect to each of 
the parameters was observed and the determination of this zenith distance to within $10 \mathrm{~cm}$ (ignoring the atmospheric propagation delay) was made. Contours showing the observed and residual signatures for each of these experiments are shown in Figure 10. Again, note that the amplitude and parabolic shape of the transponder return clearly identify it in the observed signatures.

\section{GALCULATION OF DISTANCE FROM ALTIMETER TO TRANSPONDER}

According to the European Space Agency, the pre-set component of the waiting time for the 7 July 1993 overpass was

$$
\begin{aligned}
(392160+33309.113281 & -2496) \times 12.5 \mathrm{~ns}-29.8 \mathrm{~ns} \\
& =5287134.116 \mathrm{~ns}
\end{aligned}
$$

in order to produce a spike located in bin 32 . The first term $392160 \times 12.5 \mathrm{~ns}$ and the third term $-2496 \times 12.5 \mathrm{~ns}$ are fixed values; the middle term is the delay set by the recent history of the returns.

Since this is the expected delay for a pulse to be transmitted and returned, the one-way distance is

$$
5287134.116 \times 10^{-9} \times \frac{c}{2}=792521.466 \mathrm{~m} .
$$

The best fit of the theoretical signature to the observed signal places the zenith-most return at bin 22.717 , i.e. 9.283 bins before bin 32 ; since the duration $B$ of each bin is $12.159533 \mathrm{~ns}$, this is a decrease in the one-way distance by an amount

$$
9.283 \times 12.159533 \times 10^{-9} \times \frac{c}{2}=16.920 \mathrm{~m}
$$
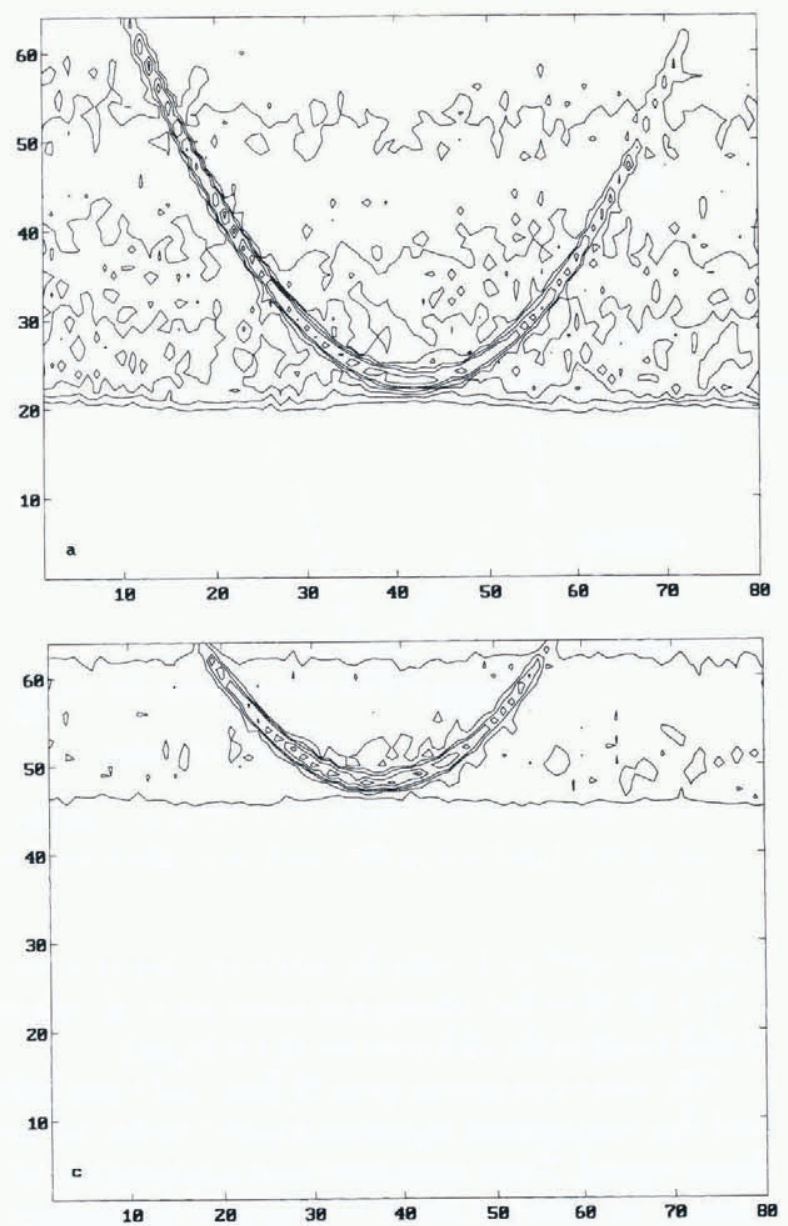

Fig. 10. Contour of the observed signature of the transponder obtained ( a) on 30 June 1995 and (c) on 1 July 1995, and contours of the residual signatures after the removal of the best-fitting transponder return $(b, d)$. for a total one-way travel distance from the altimeter to the effective point of reflection of the transponder that is $D_{\mathrm{TA}}=792521.466-16.920=792504.546 \mathrm{~m}$.

A "front-end measurement" (Francis, 1993) contributes an external range bias of $\mathrm{d} R=-41.5 \mathrm{~cm}$ which must be subtracted from the measured range, giving a final range of $792504.961 \mathrm{~m}$.

Equivalent calculations for the experiments of 30 June 1995 and 1 July 1995 give the altimeter-transponder distances at these instants as 792553.673 and $792564.188 \mathrm{~m}$, respectively.

We note that atmospheric corrections must be taken into account in order to determine this final distance accurately.

\section{DETERMINATION OF DEPTH WITHIN SNOW FROM WHICH FIRST REFLECTION OCCURS}

In each transponder experiment, the location of the site was carefully selected. The position was near the GRIP camp in a well-surveyed region known to have a low surface slope, minimal sastrugi and dry, cold snow. The transponder was then placed at a point directly below a predicted crossover, and the exact location determined by a global positioning system instrument.

For 7 July 1993 the location was determined as $72.45897650^{\circ} \mathrm{N}, 39.01542036^{\circ} \mathrm{W}$. This point is $1.1010 \mathrm{~km}$ from the surface track. In 1995 , the location was $72.45620830^{\circ} \mathrm{N}$, $39.018726833^{\circ} \mathrm{W}$ for both acquisitions of the signal. For the 30 June 1995 experiment with the ERS-1 altimeter, the ground track was then $0.7780 \mathrm{~km}$ from the transponder, while for the 1 July 1995 result with the ERS-2 altimeter
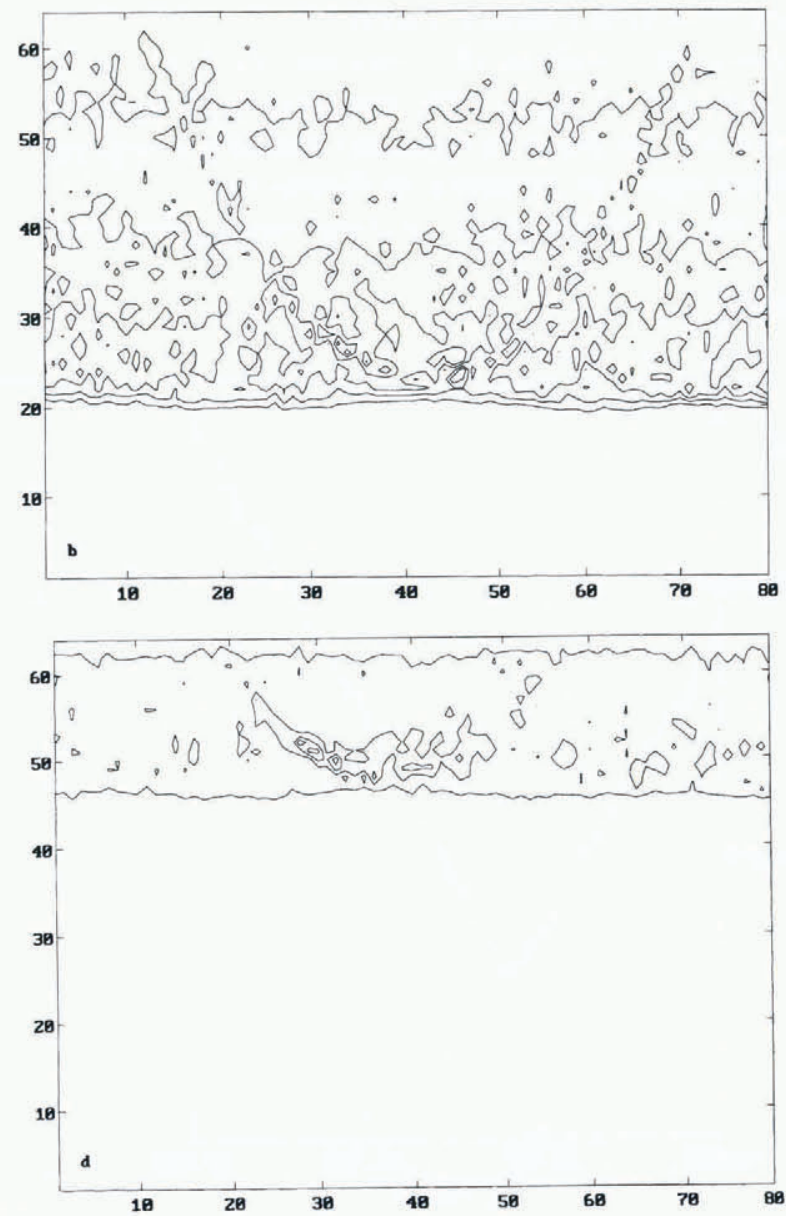
the ground track was $0.7303 \mathrm{~km}$ from the transponder. In all cases, the surface track passed between the location of the transponder and the highest point on the ice cap, so that the transponder was at a slightly lower elevation than the point directly below the satellite.

From a contour map of the area (Fig. 11; Forsberg, 1996), it is apparent that the snow surface is very regular in this region. The local slope of the surface is $1.603 \pm 0.100 \mathrm{~m} \mathrm{~km}^{-1}$, at an angle $\alpha_{0}=0.001603 \mathrm{rad}$ or $0.09185^{\circ}$ to the horizontal. The angle between the surface track of the satellite and the direction of the gradient is $\beta=138^{\circ}$.

\section{Surface Topography}

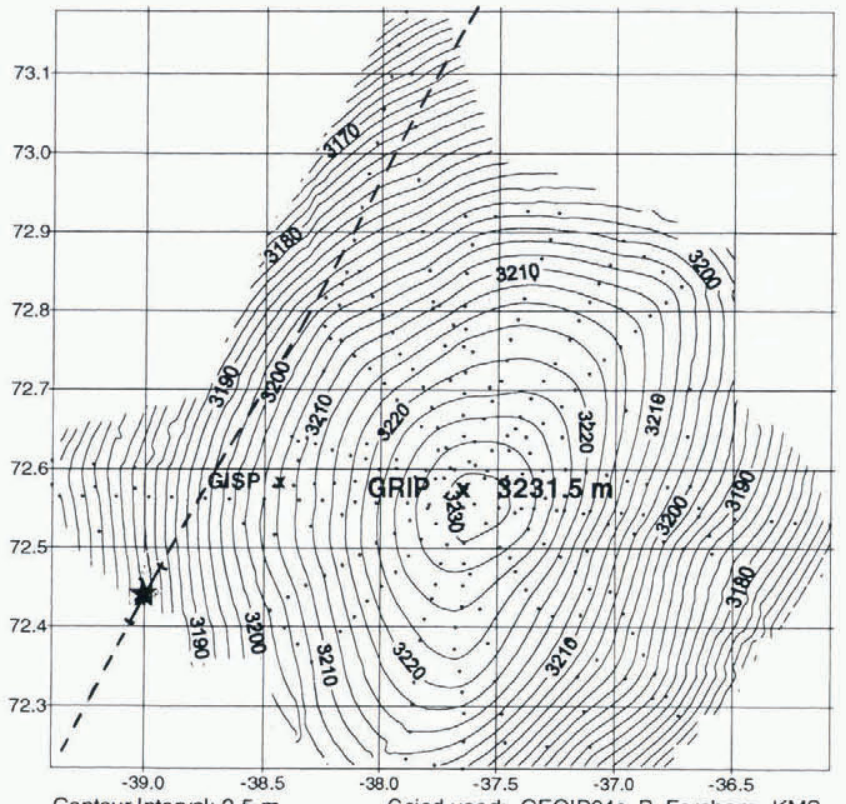

Contour Interval: $2.5 \mathrm{~m}$

$30 \mathrm{~km}$

Fig. 11. Surface topography near the GRIP site (Forsberg, 1996). Contour interval is $2.5 \mathrm{~m}$. Dashed line shows ground track of ERS-1; star locates position of transponder; short, solid segment indicates duration of orbit during which signal from transponder was acquired.

The transponder sits on the snow surface, and the top of the instrument is $0.800 \mathrm{~m}$ above the snow. The electrical delay of the transponder (the time taken to capture, amplify and transmit the return signal) is equivalent to a distance of $6.780 \pm 0.026 \mathrm{~m}$ (Rutherford Appleton Laboratory analysis), meaning that the effective point of reflection for the transponder is $3.390-0.800 \mathrm{~m}$ below the snow surface. Therefore the distance from the point on the snow surface directly below the transponder to the altimeter's zenith position, $P_{0}$, is $D=D_{\mathrm{TA}}+(-3.390+0.800) \mathrm{m}$. For the three experiments these distances are then

$$
\begin{array}{cc}
D_{\mathrm{TA}} & D \\
\mathrm{~m} & \mathrm{~m}
\end{array}
$$

$\begin{array}{lll}\text { 7 July } 1993 & 792504.961 & 792502.371 \\ \text { 30 June } 1995 & 792553.673 & 792551.083 \\ \text { 1 July 1995 } & 792564.188 & 792561.598\end{array}$

A coordinate system with the origin at the Earth's center, the $x$ axis in the direction of the surface track, and the $z$ axis through the surface track and $P_{0}$, allows the local surface height to be modeled as

$$
\begin{aligned}
z(x, y) & =\sqrt{R^{2}-x^{2}-y^{2}}+\alpha_{0}(x \cos \beta+y \sin \beta) \\
& \approx R-\frac{x^{2}+y^{2}}{2 R}+\alpha_{0}(x \cos \beta+y \sin \beta)
\end{aligned}
$$

and $P_{0}$ as $\left(0,0, R+D_{0}\right)$. In this coordinate system, the point on the snow surface directly below the transponder's location is $\left(0, y_{\mathrm{T}}, z\left(0, y_{\mathrm{T}}\right)\right)$, and the distance from this point to $P_{0}$ is

$$
D=\sqrt{y_{\mathrm{T}}^{2}+\left[R+D_{0}-z\left(0, y_{\mathrm{T}}\right)\right]^{2}} .
$$

Since $y_{\mathrm{T}}, R$ and $D$ are known, this determines the distance $D_{0}$ as

$$
D_{0}=\sqrt{D^{2}-y_{\mathrm{T}}^{2}}+\alpha_{0} y_{\mathrm{T}} \sin \beta-\frac{y_{\mathrm{T}}^{2}}{2 R} .
$$

The distance from $P_{0}$ to an arbitrary point on the snow surface is $d$ where

$$
\begin{aligned}
d^{2} & =x^{2}+y^{2}+\left[R+D_{0}-z(x, y)\right]^{2} \\
& =x^{2}+y^{2}+\left[D_{0}+\frac{x^{2}+y^{2}}{2 R}-\alpha_{0}(x \cos \beta+y \sin \beta)\right]^{2} .
\end{aligned}
$$

Then the point on this snow surface closest to $P_{0}$, from which the first return will arrive, is at $\left(x_{\mathrm{c}}, y_{\mathrm{c}}, z_{\mathrm{c}}\right)$ where $x_{\mathrm{c}}$ and $y_{\mathrm{c}}$ minimize $d^{2}$.

This gives the conditions that

$$
x_{\mathrm{c}}=r_{\mathrm{c}} \cos \beta \quad \text { and } \quad y_{\mathrm{c}}=r_{\mathrm{c}} \sin \beta
$$

where

$$
r_{\mathrm{c}}+\left(D_{0}+\frac{r_{\mathrm{c}}{ }^{2}}{2 R}-\alpha_{0} r_{\mathrm{c}}\right)\left(\frac{r_{\mathrm{c}}}{R}-\alpha_{0}\right)=0 .
$$

The equation for $r_{\mathrm{c}}$ can be solved iteratively, then $z_{\mathrm{c}}$ and finally $d$ can be determined.

$\begin{array}{ccccc}D & y_{\mathrm{T}} & D_{0} & r_{\mathrm{c}} & d \\ \mathrm{~m} & \mathrm{~m} & \mathrm{~m} & \mathrm{~m} & \mathrm{~m}\end{array}$

\begin{tabular}{llllll}
\hline 7 July 1993 & 792502.371 & -1101.0 & 792500.121 & 1129.9 & 792499.215 \\
30 June 1995 & 792551.083 & -778.0 & 792549.671 & 1130.0 & 792548.765 \\
1 July 1995 & 792561.598 & -730.3 & 792560.298 & 1130.0 & 792559.392
\end{tabular}

An average of the five waveforms (from the residual signatures) nearest to that generated when the altimeter was at $P_{0}$ was used to determine the return from the surface. Each of these average waveforms was fitted (Fig. 12) to a fiveparameter model of a "single-ramped" waveform (Zwally and others, 1994).

For the 7 July 1993 signal, the first reflection from the snow occurs at bin $19.81 \pm 0.100$, preceding the transponder return by $2.907 \pm 0.100$ bins. This corresponds to a one-way distance of $2.907 \times 1.822668=5.298 \pm 0.182 \mathrm{~m}$. The distance from the altimeter to this reflecting surface within the snow is then

$$
\begin{aligned}
x_{\mathrm{r}}=D_{\mathrm{TA}}-5.298 & =792504.961-5.298 \\
& =792499.663 \pm 0.182 \mathrm{~m} .
\end{aligned}
$$

This then implies that the first return from the snow surface occurs at a depth of $0.448 \mathrm{~m}$ in the snow. 
The depths calculated in the same manner for the three experiments are:

\begin{tabular}{lccccc} 
& $D_{\mathrm{TA}}$ & Delay & Delay & $x_{\mathrm{r}}$ & $x_{\mathrm{r}}-d$ \\
& $\mathrm{~m}$ & bins & $\mathrm{m}$ & $\mathrm{m}$ & $\mathrm{m}$ \\
\hline 7.July 1993 & 792504.961 & 2.91 & 5.298 & 792499.663 & 0.448 \\
30 June 1995 & 792553.673 & 1.87 & 3.408 & 792550.264 & 1.499 \\
1 July 1995 & 792564.188 & 1.52 & 2.770 & 792561.421 & 2.029 \\
\hline
\end{tabular}

The two vertical lines in each diagram of Figure 12 indicate the bin positions associated with our location of the snow surface and with the first return from the surface. In each case, the location of the snow surface corresponds to the left vertical line, and the first return location corresponds to the right vertical line.

In this analysis of the location of the surface of first reflection in the snow, it is important to note that atmospheric corrections that are necessary in the determination of the actual distance between the transponder and the altimeter are not required here. Since exactly identical atmospheric corrections will apply to the transponder return and the reflection from the snow surface, and we are essentially dealing with the difference between these two travel times, the two corrections will cancel each other.

\section{ERROR ANALYSIS}

The distance, $D_{\mathrm{TA}}$, obtained from fitting the theoretical signature to the observed signature has an estimated error of 0.010 bins or $0.018 \mathrm{~m}$ (see Figs 6-9). The distance between the transponder and the surface track, $y_{\mathrm{T}}$, has an estimated error of $\pm 0.1 \mathrm{~m}$, and the angle $\beta$ has an error of $\pm 2^{\circ}=$ $\pm 0.035 \mathrm{rad}$.

The error estimate then computed for $D$ is $\pm 0.024 \mathrm{~m}$ and for $D_{0}$ is $\pm 0.090 \mathrm{~m}$. Then the error in $r_{\mathrm{c}}$ is $\pm 70.5 \mathrm{~m}$, in $z$ is $\pm 0.160 \mathrm{~m}$ and in $d$ is $\pm 0.208 \mathrm{~m}$.

The location of the first reflection from the snow, from the waveforms is estimated to have an error of \pm 0.100 bins or $\pm 0.182 \mathrm{~m}$, and hence the error estimate on the depth within the snow at which the first reflection occurs is estimated to have an error of $\pm 0.276 \mathrm{~m}$. We consider these error estimates conservative; the differences between the results in 1993 and 1995 are considerably larger than three times this standard deviation. A possible cause of these differences is the snow conditions at the times of the measurements.

\section{CONCLUSION}

Because of the unique nature of the transponder return, it can be clearly identified in the altimeter record and then can be modeled very well, leaving the return from the snowfield. The separation of the total signature into its transponder and snowfield components is excellent. The modeling is very sensitive to the transponder parameters and varies smoothly with these parameters, resulting in an accuracy below the $10 \mathrm{~cm}$ level (ignoring the atmospheric propagation delay). The first reflection of the radar pulse from the compact, dry snow near the top of the Greenland ice cap occurs within $2 \mathrm{~m}$ of the snow surface. The atmospheric propagation delay in the pulse's two-way transit time will have to be incorporated to determine the actual distance from the transponder to the satellite in future analyses; how-
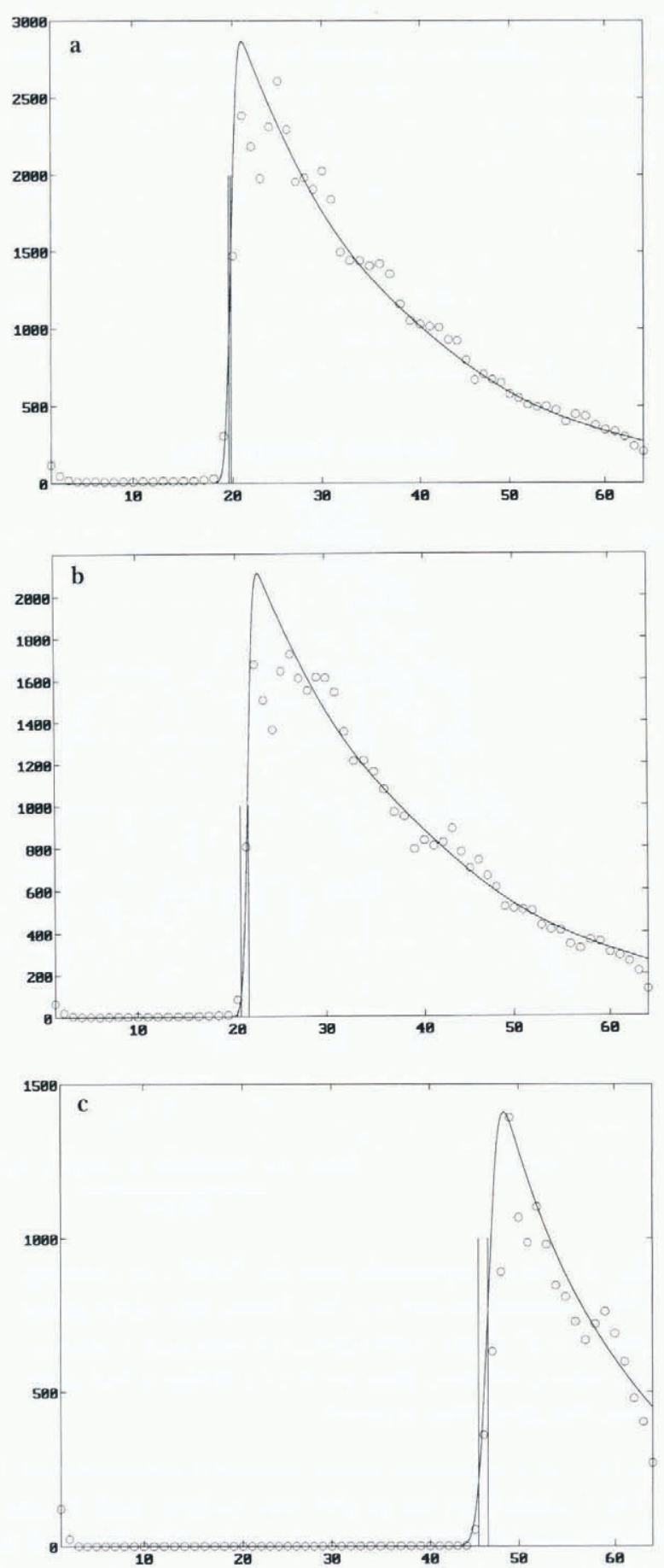

Fig. 12. Average waveform of the five waveforms when the altimeter was nearest the zenith position (open circles), and the five-parameter "single-ramped" model ( solid line) for each of the three transponder experiments: (a) 7 July 1993, (b) 30 fune 1995 and (c) 1.July 1995. Right vertical line is the bin location of the first return from the snow from the model, and left vertical line is the bin location of the snow surface.

ever, in this determination of depth within the snow at which the signal reflection occurs, this bias occurs identically to the two reflections under consideration and has no effect on the difference between these two distances.

\section{REFERENCES}

Brown, G. S. 1977. The average impulse response of a rough surface and its applications. IEEE 7. Oceanic Eng., AP-25 (1), 67-73.

Ekholm, S. and K. Keller. 1993. Gravity and GPS survey on the summit of the Greenland ice sheet 1991-1992. Copenhagen, National Survey and Cadastre. Geodetic Division. (Technical Report 6.) 
European Space Agency (ESA). 1993. ERS-l user handbook. Revision. Paris, European Space Agency. (ESA SP-1148.

Forsberg, R. 1996. The geoid of Greenland - a reference surface for remote sensing. Proceedings of the Gth workshop on mass balance of the Greenland ice sheet and related topics. Copenhagen, Geological Society of Greenland GEUS), 27-32.

Francis, C. R. 1993. The calibration of the ERS-1 radar altimeter. ESA Rep. ER-RP-ESA-RA-0257, Issue 2.0.

Jezek, K. C., P. Gogineni and M. Shanableh. 1994. Radar measurements of melt zones on the Greenland ice sheet. Geophys. Res. Lett., 21 1), 33 - 36.

Keller, K., N. Gundestrup, C. C. Tscherning, R. Forsberg and S. Ekholm. 1994. Mass balance studies at the Dome GRIP using GPS and gravity. Grönlands Geologiske Undersögelse, Open File 94-13, 66-69.

Powell, R.J. 1986. Relative vertical positioning using ground-level trans- ponders with the ERS-I altimeter. IEEE Trans. Geosci. Remote Sensing. GE-24(3), 421-425.

Powell, R. J., A. R. Birks, W. J. Bradford, C. L. Wrench and J. Biddiscombe. 1993. Using transponders with the ERS-1 altimeter to measure orbit altitude to $\pm 3 \mathrm{~cm}$. In Kaldeich, B., ed. Proceedings of the First ERS-I Symposium Space at the Service of our Environment, t-6 November 1992, Cannes, France. Paris, European Space Agency, 511-516. (ESA Special Publication SP-359.)

Zwally, H.J., A. C. Brenner, J. DiMarzio and T. Seiss. 1994. Ice sheet topography from retracked ERS-1 altimetry. In Space at the Service of our Emironment. Proceedings of the Second ERS-1 Symposium, II- It October 199.3, Hamburg, Germany. Vol. 1. Paris, European Space Agency, 159-164. (ESA SP-361.

MS received 27. November 1995 and accepted in revised form 1 fune 1998 